\title{
Real Workload-Situated Training in COVID-19 \\ Prevention of General Practice Residents in China: A Situated Cognition Study
}

\section{Xin Rao (D 617993959@qq.com )}

West China Hospital of Sichuan University

Li Luo

Affiliation囚Sichuan University

Qiaoli Su

West China Hospital of Sichuan University

Xingyue Wang

West China Hospital of Sichuan University

\section{Research Article}

Keywords: COVID-19, real epidemic situated training (REST), NASA, public health training, epidemic task.

Posted Date: October 12th, 2021

DOI: https://doi.org/10.21203/rs.3.rs-854514/v1

License: @ (i) This work is licensed under a Creative Commons Attribution 4.0 International License.

Read Full License 


\section{Abstract}

Objective:

The participation of general practice (GP) residents in COVID-19 prevention and control tasks touched workload participation in public health and disease prevention and control and was also a rare, valuable training experience for the residents and research material for medical education. This experience contributed to the understanding of three key points: First, was the content of the COVID-19 prevention task suited to them, or did it overload them in the present? Second, their competence in the COVID-19 prevention task reflected whether the early medical school training was sufficient or not .Third, what can be drawn from this study to promote public health training in the future? This study aimed to explore these issues by conducting a real epidemic situated training (REST) program.

Methods:

A situated cognition study was designed that included situational context design, legitimate peripheral participation, and the construction of a community of practice. The Task Cognitive Load Scale (NASATLX Scale) and self-developed questionnaires were adopted to conduct a questionnaire survey of resident doctors in a GP training program from West China Hospital of Sichuan University, and 183 questionnaires were collected. SPSS 23.0 statistical software was used for the statistical analysis of data.

Results:

The NASA scale showed that the intensity of field epidemic prevention and control (training) was tolerable. In particular, there was no statistical difference in the cognitive load intensity of training before and after the epidemic occurred at different time points $(P<0.05)$. This shows that they were early trained and well prepared before sudden outbreak of the COVID-19.Before the outbreak of the epidemic, the public health knowledge and training received came from undergraduate education (83.16\%), early residents program training (69.47\%), online self-study (49.16\%), and continuing education (20.53\%).

Conclusion:

Former medical school education and training at the regulatory training stage have a good effect and enable residents to master the skills required for epidemic prevention and control and to physically and mentally prepare for the task. After this stage, epidemic prevention and control training in real situations will make important contributions to the self-assessment and performance improvement of public health training,and make participants more competent to face the epidemic task.

\section{Introduction}

The sudden outbreak of the COVID-19 pandemic highlighted the importance of competent community prevention as the front line of epidemic prevention and control and obliged practitioner residents to 
become part of such teams. The involvement of residents touched workload participation in public health and disease prevention and control and was also a rare and valuable training experience.

Learning evolves within the simulation environment from the interactions of (a) activity, (b) people, and (c) prior knowledge that is brought to the situation. When knowledge and learning are theorized in terms of representations stored and processed in the mind, empirical and theoretical developments in very different scholarly disciplines have led to the emergence of the situated cognition hypothesis, which consists of a set of interlocking theses: cognition is embodied, fundamentally social, distributed, and enacted and often works without representations.[1] A number of theoretical perspectives[2-4] have suggested that learning is socially situated, and cognitive development occurs through relations between human interactions and the social or cultural context of everyday activities, thereby laying the groundwork for challenging the assumption that skills learned in the classroom can simply be transferred to a practical environment.[5] In other words, students, as newcomers, are guided by more experienced faculty and clinicians to actively take part in the learning process, which allows them to construct their own knowledge based on relationships with other learners, activities, environmental cues, and the social organization of learning events. Situated cognition has been established as a useful framework for understanding and guiding teaching and learning processes in medicine education and training .[6]

Simulation-based learning is a dynamic process that can be used to build a community of practice where learners can observe and participate in meaningful realistic encounters to build their skills and understanding.[7-9] Moreover, they can gain insights and feedforward, which is described as the opportunity to envision future activities after mastering certain skills and behaviors. $[5,10]$

The outbreak of the COVID-19 pandemic conduct a real situation for the GP resident to participate in the public health task . $[11,12]$ This real situation may be a once-in-a-century experience that cannot be replicated. The real epidemic situated training (REST) program during COVID 19 has many implications for the field public health training. This study aimed to explore the research on the training content for residents as well as their cognitive load and abilities improvement at different time points (before, during, and after the outbreak) in a real situation, to use in the study of the resident training process.

\section{Methods}

\section{Design}

A situated cognition study was designed to built a training environment and The Task Cognitive Load Scale (NASA-TLX Scale) and self-developed questionnaires were conduct, aiming to assess how their workload in this training task, how well they were prepared for the sudden epidemic and how will their performance for the future public health prevention , to implicate how well our public public health prevention training for our GP medical students and GP residents.

\section{Setting (A situated cognition study)}


Situated cognition is classified into studies of situational context design, legitimate peripheral participation, and the construction of a community of practice. [1]

\subsection{Situational Context Design}

Following the outbreak of the COVID-19 epidemic, China initiated a first-level response to the public health emergency.[13] The main purpose of standardized training is to train qualified grassroots general practices, and an important part of the training content concerns responding to public health emergencies. Community health service centers played an extremely important role as the first line of defense in the prevention and control of the COVID-19 epidemic. They also provided real training scenarios for scientists' basic epidemic prevention practices. Each grassroots practice base must be reinforced according to the epidemic prevention and control situation.The teaching, training, and practice of infectious disease prevention and control knowledge and skills will use the completed training practice as an important basis for assessment of the process.

a. Pre-vocational training (they short training the participants get before they tackle the real task ) and assessment

1. National Basic Public Health Service Standards - Service Standards for Reporting and Handling of Infectious Diseases and Public Health Emergencies

2. Knowledge of epidemic prevention and control, hospital infection prevention and control, personal protection skills, etc.

3. Introduction to each point of the institution's epidemic prevention and control work (layout, process, work content, specifications, etc.)

4. Written examination to assess knowledge of epidemic prevention and control

5. Standard patient (SP) assessment, prevention and control deployment process

b. Materials guarantee

Provide personal protective materials (such as masks, gloves, protective clothing, isolation clothing, etc.) required for training participants at different points in the work

\subsection{Legitimate Peripheral Participation}

"legal peripheral participation" means learners should enter the scene, start at the edges, and continue to advance gradually and more deeply to master the core essentials and exert their subjective initiative in practice. $[14,15]$ The acquisition of practical skills by students is a gradual process that does not occur overnight and requires the joint effort and participation of teachers and students. Online virtual 
simulation experiments can provide modular learning, and learners can complete different learning tasks according to their own learning needs.[16-18] Through activities ranging from easy to difficult, teachers can inspire or induce students to actively explore in real or virtual experimental environments to give full play to the students' subjective initiative and in-depth studies. When students has cultivated themselves with competence, support and help from teachers can be appropriately reduced. According to the prevention and control work needs, the West China Hospital of Sichuan University arranges training for trainees to rotate in various epidemic prevention and control positions in the community health center and participate in prevention and control work. In addition, it arranges special personnel to be responsible for teaching. Under the guidance of the senior physician, the resident gradually becomes familiar with the work process.

The real epidemic situated training (REST) program during COVID-19 was conducted as following.

1. Pre-inspection and triage: participate in pre-inspection and triage, become familiar with the work process and specifications, master the main points of pre-inspection (temperature measurement, epidemiological history collection, simple inquiry of clinical symptoms, full use of general practice thinking) and become familiar with the triage process (outpatient clinic, general outpatient clinic, fever clinic, and referral).

2. Medical observation of quarantined persons:

1) Home quarantine personnel: participate in the medical observation work of home quarantine personnel temperature measurement and health and travelling history inquiries.

2) Observation and isolation personnel: participants in medical observation on the personnel under centralized observation.

3) Concentrated residents: trainees of the plan can participate in the duty and medical observation of the hotels where people from Hubei (or other areas with a high rate of infection)

3, Participation in epidemic prevention and control and the daily work of the family medicine team:

1) Education on epidemic prevention and control knowledge of contracted residents conducted through online methods such as online telephone and online software.

2) Chronic disease follow-up and health management during the epidemic period

3) Practice of group epidemic prevention and elimination in enterprises, institutions, and schools

4) Prevention and control of hospital infection and practice of occupational protection

5) Other public health work in the community 
4. According to the specific situation, arrange for training students to participate in fever clinics, general clinics, children's health care, Chinese medicine, and rehabilitation and record the workload.

\subsection{Construction of a Community of Practice}

Contextual cognitive learning theory emphasizes the construction of a community of practice. Teachers are not only lecturers of knowledge, but also the promoters of knowledge, the learning partners of students, and the promoters of communication among members of the community. $[19,20]$ While teaching knowledge and cultivating abilities, experimental teaching also emphasizes the establishment of good thinking habits for students. Based on the real clinical cases, students can restore the complex context of knowledge generation and development under the guidance of teachers and obtain more flexible knowledge which adapt to the real situation. Through different case situations, students will have more opportunities to solve problems and apply the theoretical knowledge they have learned to their practice. In the community, there is interaction among individuals and between individuals and the environment. This learning process is a process of interaction, communication, and dialogue.

Participants share resources, respect, and trust one another. In the practice community, the identity of the learner is continuously reproduced, and the learner moves from being a bystander to a participant and, finally, to a demonstrator of mature practice. In other words, they change from being a legitimate marginal participant as a novice to being a core member of the community as an expert step by step.[21, 22]

As it is described above, the real situational design of REST program is drawn in Table 1

\section{Participant}

Resident doctors in a GP training program from West China Hospital of Sichuan University, and 183 questionnaires were collected. The participants were described in Table 2.

\section{Cognitive Load Scale (NASA-TLX Scale) and Questionnaires}

The NASA Task Load Index (TLX) is a popular technique for measuring subjective mental workload.[2325] It relies on a multidimensional construct to derive an overall workload score based on a weighted average of ratings on six subscales: mental demand, physical demand, temporal demand, performance, effort, and frustration level.[24, 26] The Object Cognitive Load Scale (NASA-TLX Scale) and selfdeveloped questionnaires were adopted to conduct a questionnaire survey of resident doctors who were in a GP training program in West China Hospital of Sichuan University, and 183 questionnaires were finally collected. The NASA-TLX Scale and self-developed questionnaires were finally merged into one questionnaire. The questionnaire prompts were as follows: 
1. Have you participated in community epidemic prevention work?

2. When you participated in the epidemic prevention work, your identity was [single-choice question]

3. You participated in the prevention and control of the epidemic several months after the outbreak. [single-choice question]

4. In which community health service centers did you participate in epidemic prevention and control? [multiple-choice question]

5. The main contents of your participation were [multiple-choice question]

6. The training you received before taking up your post included [multiple-choice question]

7. The teacher who provided you with pre-vocational training came from [multiple-choice question]

8. Degree of mental and cognitive stress when you participated in prevention and control work [singlechoice question]

9. When you participated in the prevention and control work, did you have a heavy mental and physical burden? [multiple-choice question]

10. When you participated in the prevention and control work, did you have a strong sense of time urgency? [multiple-choice question]

11. When you participated in the prevention and control work, how hard did you need to work compared with other work? [single-choice question]

12. Did you feel pressure or worry during your participation in the prevention and control work? [multiplechoice question]

13. What is your satisfaction score with the REST program?

14. Are you willing to participate in epidemic prevention and control and continue to receive relevant training? [multiple-choice question]

15. Before the outbreak, the public health training you received included [multiple-choice question]

16. Before the outbreak, the public health knowledge and training you received came from [multiplechoice question]

17. If you are responsible for the prevention and control of an epidemic situation in a community in the future, the level you can achieve is [single-choice question]

\section{Date analysis}


SPSS 23.0 statistical software was used for the statistical analysis of data.(Chi-square estimates, confidence interval 95\%)

\section{Results}

According to the pre-survey and statistical analysis, the Cronbach's alpha coefficient of the questionnaire was 0.91 .

\subsection{For the "Situational Context Design"}

The real situational context of REST program is drawn in Table 1 showing above.

\subsection{For the "Legitimate Peripheral Participation "}

Before the outbreak of the epidemic, the public health training received included basic knowledge of infectious diseases (81.58\%), putting on and taking off protective clothing and other aseptic operations (84.74\%), and hospital infection training (85.26\%). Before the outbreak, the public health knowledge and training received came from undergraduate education (83.16\%), early resident training (69.47\%), online self-study (49.16\%), and continuing education (20.53\%).

In the REST program , more than $60 \%$ indicators of the GP residents' cognitive load on the NASA scale were at a "moderate" level, indicating that the physical and mental workload of the GP residents in the REST program during COVID 19 was at an acceptable level, and the training content, ability improvement, and cognitive load were also fair. In other words, as a GP residents, they were well prepared for such a pandemic outbreak.

For the questionnaire, The NASA scale showed is as following Table3 .This generally indicated that the intensity of field epidemic prevention and control (training) was tolerable. This also showed that participants were well trained, prepared, and qualified, with public health knowledge and skills from their former medical schools and through other relevant paths.

There was no statistical difference in the cognitive load intensity of training before and after the epidemic occurred at different time points $(P<0.05)$, which also shows that the previous and pre-vocational training was relatively sufficient and adapted well to the public health emergency and the epidemic.

\subsection{For the "Construction of a Community of Practice"}

"Construction of a Community of Practice" call for sociality situatedness. In the REST program, resident doctors who provided pre-vocational training for prevention and control included undergraduate teachers, 
hospital-related department teachers, community-based teachers, grassroots government, community staff and hotel staff, senior resident students and other teachers, indicating that a network was initially formed for epidemic prevention and control.

To determine how well resident doctors would perform if responsible for managing public health disease prevention and control, they were asked, "How well would you perform if you took on the responsibility of prevention and control of an epidemic situation in a community in the future?" The answers were divided into five levels:

- Mid-level: the participants could take on responsibility with guidance and supervision (50\%)

- Fair: the participants could accomplish it independently (24.74\%)

- Well: the participants could instruct junior or other students $(11.58 \%)$

- Very well: the participants would have more constructive suggestions (4.74\%)

- Unsure: $8.95 \%$

Different supervision reflects different relevant learning performance outcomes.[28-30]

\section{Discussion}

Situated learning theory concerns the characteristics of learning, such as sociality situatedness, complexity, and competence. Situated learning theory has expanded the nature of learning, which is "from getting to participate." Legitimate peripheral participation provides an innovative analytical insight into medical education. The sudden outbreak of the COVID-19 pandemic highlighted the importance of competent community,creating a real worked loaded public health training experience for the GP residents and cognitive load of residents to reveal training implications for how well we do just before the COVID -19 pandemic, during the COVID -19 pandemic and in the future $[6,31,32]$. From the study ,the REST training mode can be applied in future circumstances.

The situated learning theory provide the medical education research a framework to design and make assessment for one particular learning content . However, sudden outbreak of the COVID-19 has created a real nature circumstances for public health program design and assessment. This study can be applied to other further study in public health education.

\section{Conclusion}

Former medical school education and training at the regulatory training stage have a good effect and enable residents to master the skills required for epidemic prevention and control and to physically and mentally prepare for the task. After this stage, epidemic prevention and control training in real situations will make important contributions to the self-assessment and performance improvement of public health training. 


\section{Advantages Of The Study}

The sudden outbreak of the COVID-19 pandemic was a rare occurrence in history that allowed for a unique study case. This situated cognition study was naturally designed in a workload training situation in real disease prevention circumstances and included situational context design, legitimate peripheral participation, and construction of a community of practice, which made it unique among other situated cognition studies. The Object Cognitive Load Scale (NASA-TLX scale) were applied to measure the public health training outcome, especially before the COVID -19 pandemic, during the COVID -19 pandemic and in the future.

\section{Limitations Of The Study}

Further study need to be followed-up, especially regression discontinuity design(RDD) research method, to reveal the effect of public health prevention training at different stages of the COVID-19 pandemic.

\section{Abbreviations}

GP: general practice

\section{Declarations}

\section{Ethical Approval}

The studies involving human participants were reviewed and approved by West China Hospital Ethics Committee (2020YFQ0011, Sichuan, China). Written informed consent was obtained from the participants of the study.

\section{Consent to Publish}

No potentially identifiable human images or data were presented in this study.

\section{Availability of Data and Materials}

The data in this manuscript are available by contacting the author.

\section{Conflict of Interest}

The authors declare that the research was conducted in the absence of any commercial or financial relationships that could be construed as a potential conflict of interest. 


\section{Authors' Contributions}

All authors participated in the design of the study and contributed to the drafting of the paper. RX designed the research and was major contributor in writing the manuscript. LL and SQ guided the discussion parts and WX discussed the result. All authors read and approved the final manuscript.

\section{Funding}

This work was supported by the Funds: Science and Technology Department Project "Integrated health management and family doctor contract service" (2020YFQ0011, Sichuan, China). The study funder aims to improve health conditions in China and make progress in medical education and training.

\section{Acknowledgments}

We would like to acknowledge the Health Commission of Sichuan Province for supporting this research program.

\section{References}

1. Roth, W.M. and A. Jornet, Situated cognition. Wiley Interdiscip Rev Cogn Sci, 2013. 4(5): p. 463-478.

2. Artino, A.R., Jr., It's Not All in Your Head: Viewing Graduate Medical Education Through the Lens of Situated Cognition. J Grad Med Educ, 2013. 5(2): p. 177-9.

3. Daniel, M., et al., Situated cognition: clinical reasoning and error are context dependent. Diagnosis (Berl), 2020. 7(3): p. 341-342.

4. Rencic, J., et al., A situated cognition model for clinical reasoning performance assessment: a narrative review. Diagnosis (Berl), 2020. 7(3): p. 227-240.

5. Smeding, A., et al., Tracking and simulating dynamics of implicit stereotypes: A situated social cognition perspective. J Pers Soc Psychol, 2016. 111(6): p. 817-834.

6. Rencic, J., et al., Clinical reasoning performance assessment: using situated cognition theory as a conceptual framework. Diagnosis (Berl), 2020. 7(3): p. 241-249.

7. Vogel, D.H.V., et al., The Temporality of Situated Cognition. Front Psychol, 2020. 11: p. 546212.

8. Trentini, B., Immersion as an embodied cognition shift: aesthetic experience and spatial situated cognition. Cogn Process, 2015. 16 Suppl 1: p. 413-6.

9. Seamon, D., Situated cognition and the phenomenology of place: lifeworld, environmental embodiment, and immersion-in-world. Cogn Process, 2015. 16 Suppl 1: p. 389-92.

10. Schilhab, T. and G.L. Esbensen, Socio-Cultural Influences on Situated Cognition in Nature. Front Psychol, 2019. 10: p. 980. 
11. Ung, C.O.L., Community pharmacist in public health emergencies: Quick to action against the coronavirus 2019-nCoV outbreak. Res Social Adm Pharm, 2020.

12. Zhang, H., Early lessons from the frontline of the 2019-nCoV outbreak. Lancet, 2020.

13. Zhao, S., et al., Quantifying the association between domestic travel and the exportation of novel coronavirus (2019-nCoV) cases from Wuhan, China in 2020: A correlational analysis. J Travel Med, 2020.

14. Barragan-Iglesias, P., et al., Participation of peripheral $P 2 Y 1, P 2 Y 6$ and $P 2 Y 11$ receptors in formalininduced inflammatory pain in rats. Pharmacol Biochem Behav, 2015. 128: p. 23-32.

15. Blitzer, A. and B. Huebner, Tool use as situated cognition. Behav Brain Sci, 2012. 35(4): p. 221-2.

16. Barnett, S., et al., A Virtual Community of Practice for General Practice Training: A Preimplementation Survey. JMIR Med Educ, 2016. 2(2): p. e13.

17. Barnett, S., et al., Implementing a virtual community of practice for family physician training: a mixed-methods case study. J Med Internet Res, 2014. 16(3): p. e83.

18. Barnett, S., et al., Usefulness of a virtual community of practice and web 2.0 tools for general practice training: experiences and expectations of general practice registrars and supervisors. Aust J Prim Health, 2013. 19(4): p. 292-6.

19. Catzikiris, N., et al., Maintaining capacity for in-practice teaching and supervision of students and general practice trainees: a cross-sectional study of early career general practices. Aust Health Rev, 2018. 42(6): p. 643-649.

20. Cobb, C.L., et al., Associations among the advisory working alliance and research self-efficacy within a relational-efficacy framework. J Couns Psychol, 2020. 67(3): p. 361-370.

21. Sturman, N., M. Parker, and C. Jorm, Clinical supervision in general practice training: the interweaving of supervisor, trainee and patient entrustment with clinical oversight, patient safety and trainee learning. Adv Health Sci Educ Theory Pract, 2021. 26(1): p. 297-311.

22. Sturman, N., et al., Good help: a model for providing in-consultation supervision of general practice trainees. Educ Prim Care, 2021. 32(2): p. 104-108.

23. Ruiz-Rabelo, J.F., et al., Validation of the NASA-TLX Score in Ongoing Assessment of Mental Workload During a Laparoscopic Learning Curve in Bariatric Surgery. Obes Surg, 2015. 25(12): p. 2451-6.

24. Noyes, J.M. and D.P. Bruneau, A self-analysis of the NASA-TLX workload measure. Ergonomics, 2007. 50(4): p. 514-9.

25. Cao, A., et al., NASA TLX: software for assessing subjective mental workload. Behav Res Methods, 2009. 41(1): p. 113-117.

26. Mansikka, H., K. Virtanen, and D. Harris, Comparison of NASA-TLX scale, modified Cooper-Harper scale and mean inter-beat interval as measures of pilot mental workload during simulated flight tasks. Ergonomics, 2019. 62(2): p. 246-254. 
27. Yurko, Y.Y., et al., Higher mental workload is associated with poorer laparoscopic performance as measured by the NASA-TLX tool. Simul Healthc, 2010. 5(5): p. 267-71.

28. Stensrud, T.L., T.A. Mjaaland, and A. Finset, Communication and mental health in general practice: physicians' self-perceived learning needs and self-efficacy. Ment Health Fam Med, 2012. 9(3): p. 2019.

29. Finomore, V.S., Jr., et al., Viewing the workload of vigilance through the lenses of the NASA-TLX and the MRQ. Hum Factors, 2013. 55(6): p. 1044-63.

30. Morgan, S., et al., Test result audit and feedback (TRAFk) as a supervision method for rational test ordering in general practice training. Aust Fam Physician, 2016. 45(7): p. 518-22.

31. Morrison, J., et al., Perceptions of ad hoc supervision encounters in general practice training: $A$ qualitative interview-based study. Aust Fam Physician, 2015. 44(12): p. 926-32.

32. Rao, X., et al., The Development of a Competency Assessment Standard for general practices in China. Front Public Health, 2020. 8: p. 23.

\section{Tables}

Due to technical limitations, Tables are only available as a download in the Supplemental Files section.

\section{Supplementary Files}

This is a list of supplementary files associated with this preprint. Click to download.

- Table1TherelationshipbetweensituatedcognitiontheoryandtheRESTprogram.xlsx

- Table2participants.xlsx

- Table3TaskCognitiveLoadDistributionFromNASAScaleTable.xlsx

- originalmaterial.xIsx 